\title{
Übersicht über fremde Gesetze und Gesetzentwürfe.
}

In den letzten Jahren haben die meisten Länder sich teils neue Strafgesetze gegeben, teils mit den Arbeiten zu solchen begonnen, und fast iberall, wohin man den Blick wendet, wird man daher neue Gesetze oder wenigstens Gesetzentwürfe finden. Da eine Reihe von diesen bei der Ausarbeitung dieses Entwurfes in größerem oder geringerem Umfang benutzt worden ist, dürfte es zweckmäßig sein, ein Verzeichnis und eine kurze Darstellung der ausländischen, insbesondere europäischen Strafgesetzgebung vorauszuschicken (vgl. Die Strafgesetzgebung der Gegenwart I, 1894).

I. Schwedisches Strafgesetz vom I6. Februar I864 mit seinen Abänderungen durch die Verordnung vom 24. Mai und I9. Juli 1872, 31. Oktober 1873, r6. Juni 1875, 30. Dezember 1876, ro. August 1877, 6. August r881 und 6. Oktober r882, die Gesetze vom r6. Mai r884, 28. Oktober 1887,7 . Juni 1889 und 20. Juni 1890 , welch letzteres hauptsächlich eine Abänderung der Strafdrohungen enthält, sowie das Gesetz vom 14. Oktober 1892.

2. Dänisches Strafgesetz vom 10. Februar 1866 nebst Abänderungen durch die Gesetze vom 25. Februar 1871, 23. Mai 1873, ro. April 1874 und 9. April r89r. Vgl. auch das Strafgesetz von Island vom 25 . Juni 1869 .

3. Finnländisches Strafgesetz vom rg. Dezember 1889 nebst Abänderungen durch die Vo. vom 2 r. April r 894. Der erste Entwurf (von 1875) wurde von einer Kommission ausgearbeitet, deren wichtigstes Mitglied Prof. Ehrström war und er wurde später durch eine Prtifungskommission durchgesehen (Helsingfors 1884). Eine eingehende Kritik des Entwurfes enthält Hagströmer: Granskning usw. Upsala $\mathbf{r} 884$.

4. Das deutsche Strafgesetz vom 3 r. Mai r 87o. Dieses Gesetz, welches jedoch im wesentlichen eine Revision des preußischen von I $85 \mathbf{I}$ ist, wurde in sehr kurzer Zeit zustande gebracht, indem nämlich 
mit den Arbeiten dazu erst in der letzten Hälfte des Jahres I 868 begonnen wurde. Zusätze und Abänderungen sind gemacht worden durch die Gesetze vom 10. Dezember 1871, 23. November 1874, 6. Februar 1875 , 26. Februar 1876 , 24. Mai 1880, 5. April 1888, 13. Mai 1891, 26. März, 19. Juni und 3. Juli 1893, wozu noch zu bemerken ist, daß die Bestimmungen uber den Bankrott durch das Konkursgesetz vom ro. Februar 1877 abgelöst sind. Auferdem ist zu erwähnen das Aktien-Gesetz vom 18. Juli 1884 , das eine Reihe einschlägiger Straf bestimmungen enthält, sowie das Sprengstoffgesetz vom 9. Juni 1884 und die Seemannsordnung vom 27 . Dezember 1872.

5. Der österreichische Entwurf zu einem neuen Strafgesetz wurde erstmals der Volksvertretung im Jahre 1874 vorgelegt, er ist aber noch nicht verbeschieden. Der im folgenden zitierte Entwurf ist der von einem Ausschuß des österreichischen Reichsrats 1893 angenommene ( 709 der Beilagen zu den stenographischen Protokollen des Abgeordnetenhauses - XI. Session 1893). Das geltende österreichische Strafgesetz ist vom 27. Mai $185_{2}^{2}$, aber dieses ist wiederum zunächst nur eine Revision des Gesetzes vom 3. September 1803 .

Ein Spezialgesetz, betreffend unredliche Handlungsweise bei Kreditgeschäften wurde am 28. Mai $188 \mathrm{I}$ in 17 Paragraphen erlassen, ein solches bezüglich der Sprengstoffe am 27. Mai x 885, über Zwangsarbeitsanstalten u. a. am 24. Mai 1885.

6. Das ungarische Strafgesetz von 1878 . Deutsche Übersetzung von Steinbach, Budapest 1878 . Das ungarische Strafgesetz bezüglich Versehen von 1879 . Beiden Gesetzen liegt der Entwurf von Karl Csmegis zugrunde.

7. Der kroatische Strafgesetzentwurf. Der Entwurf ist im Laufe von $z$ wei Jahren durch den Justizchef Derenčin ausgearbeitet worden und war fertig am Ende des Jahres 1879; auf deutsch tibersetzt von Cuculic sowie in Tauffers, Gesammelte Wohlmeinungen uber den kroatischen Strafgesetzentwurf, Wien $\mathbf{x} 882$.

8. Die schweizerische Strafgesetzgebung. Die geltenden Gesetze sind: die von Thurgau 1841, revidiert 1868, Waadt von 1843, Graubünden 1851 und Polizeistrafgesetz von 1873 , Aargau von 1857 und Polizeistrafgesetz von 1868 , Wallis von 1858 , Schaffhausen von 1859 , Luzern von 1860 und Polizeistrafgesetz von 1861 , Unterwalden o. d. W. von 1864 und Polizeistrafgesetz von 1870 , Bern von 1866 , Glarus von 1867 , Freiburg von 1868 , Zürich von 187 I, Basel-Stadt 
von 1872 nebst Polizeistrafgesetz von 1872 , Basel-Land von 1873 , Tessin von 1873, Genf von 1874, Zug von r876, Appenzell a. Rh. von 1878 , Schwyz von $r 881$, Solothurn von 1885 , St. Gallen von r 885 und Neuchâtel von r89r, wozu noch das Bundesstrafgesetz von I 853 über einzelne Gebiete hinzukommt. Eine gesammelte, systematisch geordnete Ausgabe der geltenden schweizerischen Strafgesetze findet sich bei Carl Stooß. Die schweizerischen Strafgesetzbücher zur Vergleichung zusammengestellt, Basel und Genf 1890 . Vgl. von demselben: Die Grundzüge des schweizerischen Strafrechts I und II, Basel und Genf 1892 und 1893. Diese Arbeiten, die beide auf Veranlassung des Bundesrats herausgegeben sind, sind Vorarbeiten zu dem neuen von Stooß ausgearbeiteten Entwurf zu einem gemeinsamen Strafgesetz für die Schweiz. Ein vorläufiger Entwurf zum allgemeinen Teil desselben wurde nebst Motiven im Jahre 1893 veröffentlicht und ist durch eine von Juristen der verschiedenen schweizerischen Kantone zusammengesetzten Kommission durchgesehen worden.

Der Entwurf desselben Verfassers zu dem ganzen Strafgesetz nebst utbrigens sehr kurzen Motiven erschien 1894 (Schweizerisches Strafgesetzbuch, Vorentwurf, Bern 1894 ; vgl. v. Lilienthal in der Zeitschr. f. d. ges. Strafrechtsw., Bd. XV, S. 97 und 200, und Lammasch in der Zeitschr. für Schweizer Strafrecht 1894, S. 44; vgl. auch die norwegische Rechtszeitschr. f. I895, S. 305, wo eine mit Anmerkungen versehene Übersetzung des allgemeinen Teils sich befindet. ${ }^{1}$ )

9. Das niederländische Strafgesetz vom 3. März 188r. Die im Jahre 1870 eingesetzte Kommission beendete ihr Werk im Jahre 1875 , aber erst im Jahre 1879 legte die Regierung den Entwurf den Generalstaaten vor. Das Gesetz trat erst am 1. September 1886 in Kraft (Gesetz über das Inkrafttreten des Strafgesetzes vom 15. April I886), nachdem verschiedene Abänderungen in der übrigen Gesetzgebung vorgenommen worden waren, und es wurde schon vorher in einzelnen Punkten durch ein Gesetz vom 15. Januar 1886 abgeändert.

ro. In Frankreich ist es bisher auf dem Gebiete der Strafgesetzgebung ziemlich ruhig gewesen. Jedoch sind zu erwähnen, das

1) Vorentwurf zu einem Schweizerischen Strafgesetzbuch nach den Beschlussen der Expertenkommission, Bern 1896 ist zu spät in die Hănde der Kommission gelangt, als daß er noch hätte berücksichtigt werden können. 
Recidivistgesetz vom 27. Mai 1885, das Preßgesetz vom 29. Juli I 881, die Gesetze vom 24. Juli 1889 zur Beschutzung von Kindern, vom 23. Januar 1873 zur Bekämpfung der Trunksucht sowie das durch die Ermordung des Präsidenten Carnot veranlaßte Gesetz vom 28. Juli 1894 bezüglich die Bestrafung von Aufforderungen zu oder Verteidigung von gewissen gesellschaftsgefährlichen Verbrechen. I884 brachte indessen Charles Lucas beim Senat eine Petition tiber die Notwendigkeit einer Revision des Code Pénal ein. Nachdem eine Kommission sich hierfür ausgesprochen hatte, wurde 1887 vom Justizministerium eine Kommission zur Vorbereitung einer Revision der ganzen Strafgesetzgebung eingesetzt. Diese Kommission, deren Vorsitzender Ribot war, hat einen Entwurf zum allgemeinen Teil des Gesetzes ausgearbeitet. (S. Mitteilungen der intern. krim. Vereinigung Bd. IV., S. 165.)

II. Das belgische Strafgesetz vom 8. Juni 1867 (eine Revision des Code Pénal). Wichtige Bestimmungen über die Landstreicherei und den Bettel sowie die Behandlung verbrecherischer Kinder enthält das Gesetz vom 27. November 189r. Ferner sind zu erwähnen ein Sprengstoffgesetz vom 15. Oktober 1881 und ein Gesetz gegen die Trunksucht vom August 1887 .

1 2. Spanischer Strafgesetzentwurf von 1884. Ausgearbeitet von dem damaligen Justizminister Silvela in 665 Paragraphen, aber fast gänzlich ohne Motive (vgl. Gerichtssaal 1885, S. 417). Später ist indessen die Reform stehen geblieben.

13. Das geltende portugiesische Strafgesetz vom 16 . September 1886 ist im wesentlichen eine Revision des im Jahre $185^{2}$ gegebenen Strafgesetzes, das von einer 1845 eingesetzten Kommission ausgearbeitet war.

14. Das italienische Strafgesetz vom 30. Juni 1889. Der erste Entwurf wurde dem Senate während der Session $1873 / 1874$ vorgelegt. Später wurden wiederholt Vorschläge gemacht, nachdem der Entwurf unter den verschiedenen Ministerien einzelne Abänderungen erlitten hatte. Das erste Buch wurde auch von der Deputiertenkammer 1877 angenommen, aber die Annahme des Gesetzes scheiterte lange daran, daß keine Einigkeit uber die Frage zu erzielen war, ob die Todesstrafe in das neue Gesetz aufzunehmen sei. An das Strafgesetz knüpft sich das Gesetz über die öffentliche Sicherheit vom 30. Juni $₫ 889$. 
15. Der russische Strafgesetzentwurf ist von einer 1881 eingesetzten Kommission ausgearbeitet worden, unter deren Mitgliedern Tagantsew, Nekljudof und Foinitski zu nennen sind. Der Entwurf ist mit sehr ausführlichen Motiven versehen, die hauptsächlich von Tagantsew und Foinitski ausgearbeitet sind (eine deutsche Übersetzung nebst Auszügen aus den Motiven ist von Prof. Gretener besorgt).

16. Der bulgarische Strafgesetzentwurf ist vom Jahre 1888 , er ist bestimmt, das hier im wesentlichen noch geltende türkische Strafgesetz von 1857 abzulösen.

I7. Das rumänische Strafgesetz von 1864 hat bisher nur unwesentliche Veränderungen im Jahre 1874 erhalten.

18. Das serbische Strafgesetz von 1860 beruht zunächst auf dem preußischen von $185 \mathrm{I}$.

19. Das griechische Strafgesetz von 1834 ist im wesentlichen noch in Kraft, wenn auch einzelne Teile desselben später noch abgeändert sind.

20. Der englische Strafgesetzentwurf ist 1877 von James Stephen verfaßt und dem Parlament 1878 vorgelegt worden. Später ist er immer und immer wieder von den verschiedenen Ministerien dem Parlament vorgelegt worden, ohne indes zu endgültiger Behandlung gelangt $\mathrm{zu}$ sein.

Indessen hat man eine Reihe von speziellen Strafgesetzen zustande gebracht:

Sprengstoffgesetz (Explosive Substances Act) von 1883 (Zeitschr. f. d. ges. Strafrechtsw. III, 550, IV, I 23 ).

Criminal Law Amendement Act 1885 gerichtet gegen Kuppelei, Mädchenhandel usw. (s. Zeitschr. f. d. ges. Strafrechtsw.) r 886, 288.

Probation of First Offenders Act von 1887 , das ein System einführt, das dasselbe erreichen soll wie die Gesetze des Kontinents uiber bedingte Strafurteile.

The Penal Servitude Act von $189 \mathrm{r}$, welcher das Minimum der Strafarbeit auf drei (statt fünf) Jahre festsetzt.

Prevention of Cruelty against Children Act von 1889, der Maßregeln gegen die physische und moralische Verwahrlosung von Kindern enthält (vgl. Zeitschr. f. d. ges. Strafrechtsw. XI, S. 425).

Schon im Jahre 1860 erhielt Britisch-Indien sein besonderes Strafgesetz, zunächst nach einem von Lord Macaulay schon 1834 bis I 838 ausgearbeiteten Entwurf. Dieses Gesetz, das für besonders gut 
gehalten wird, "kann als das englische Strafgesetz, befreit von seinen Formalitäten und Überflüssigkeiten, systematisch geordnet und in einzelnen Teilen den Eigentümlichkeiten Britisch-Indiens abgepaßt, angesehen werden « (Stephen, History of Crim. Law. I, S. 300).

21. I 892 erhielt Kanada ein neues Strafgesetz, bestehend aus 532 Paragraphen.

22. Strafgesetz für den Staat New York vom 26. Juli r88r. Die Arbeit wurde eigentlich schon 1857 begonnen, ist aber im wesentlichen nur eine Kompilation, die auch die englische Ausdrucksweise in der Weitläufigkeit behalten hat. Eine deutsche Übersetzung derselben findet sich als Beilage in der Zeitschr. f. d. ges. Strafrechtsw. I 884 .

23. Das mexikanische Strafgesetz vom 7. Dezember 1871 in I 150 Artikeln nebst Einführungsgesetz gleichen Datums (eine deutsche Übersetzung ist als Anlage zur Zeitschr. f. d. ges. Strafrechtsw. r 894 gedruckt). 